\title{
Operational Monitoring of Rainfall over the Arno River Basin Using Dual-Polarized Radar and Rain Gauges
}

\author{
E. GORGUCCI AND G. SCARCHILLI \\ Istituto di Fisica dell'Atmosfera (CNR), Rome, Italy \\ V. CHANDRASEK AR \\ Colorado State University, Fort Collins, Colorado
}

(Manuscript received 22 February 1995, in final form 14 November 1995)

\begin{abstract}
Reflectivity $\left(Z_{H}\right)$ and differential reflectivity $\left(Z_{\mathrm{DR}}\right)$ measurements collected by Polar $55 \mathrm{C}$ over the Arno River basin in Italy are presented. The applicability of dual-polarization $\left(Z_{\mathrm{DR}}\right)$-based rainfall algorithms at $\mathrm{C}$ band in an operational setting is studied in conjunction with a network of rain gauges. Conventional pointwise comparison of radar and rain gauge estimates of rainfall, as well as statistical comparison of dual-polarization radar and rain gauge data via probability matching procedure, are presented. Error structure of reflectivity rainfall $Z-R$ relation, as well as $Z_{\mathrm{DR}}$-based algorithms, is evaluated as a function of spatial and temporal averaging. Pointwise comparison, as well as statistical evaluation based on cumulative distribution function (CDF) matching, are used to show that in an operational environment with excessive ground-clutter contamination and attenuation problems the dual-polarization-based rainfall algorithm performs better than any arbitrary $Z-R$ relation. In addition, it is shown that a dual-polarization $\left(Z_{\mathrm{DR}}\right)$ algorithm obtained matching the CDFs performs better than the best possible $Z-R$ relation.
\end{abstract}

\section{Introduction}

Remote estimation of rainfall using multiparameter radars is a topic of active research. In the last decade, several experiments have been done with polarization diversity radars with the objective of rainfall estimation. Several carefully conducted experiments have shown that polarimetric rainfall algorithms using differential reflectivity $\left(Z_{\mathrm{DR}}\right)$ can improve upon the conventional algorithms based on reflectivity alone $(Z-R$ relations), especially in absence of a good $Z-R$ equation to use (Seliga and Bringi 1976; Aydin et al. 1990; Gorgucci et al. 1995b). The experiments referred to here were carefully done in a "research mode" of operation with low-elevation scans, clear of ground clutter, and at S-band frequencies so that there is no significant attenuation. However, for operational applications fairly robust performance is needed. This paper studies the application of polarimetric radar for rainfall measurements collected under operational environments with ground-clutter contamination, with presence of attenuation and routine full $360^{\circ}$ azimuthal scans independent of the location of the storms with

Corresponding author address: Dr. Eugenio Gorgucci, Istituto di Fisica dell'Atmosfera (CNR), P.le Luigi Sturzo, 31, 00144 Rome, Italy.

E-mail: gorgucci@radar.ifa.rm.cnr.it respect to the radar. This paper presents one of the first datasets of its kind, namely, operational monitoring by a dual-polarized C-band radar over a rain gauge network; results of operational monitoring of the Arno River basin (near Florence, Italy) by the C-band dualpolarization radar, Polar 55C, are shown.

The Arno River has a history of flooding the city of Florence and the surrounding areas, damaging not only private property but also the unique priceless art treasures kept in museums and churches. This has resulted in efforts to monitor routinely the Arno River basin to obtain the cumulative rainfall over the basin with the objective of the flood forecasting, via several initiatives. Some of those include the "Arno Project" administered by National Group for Defence from Hydrogeological Hazards (CNR, Italy) and the European Economic Community (EEC) program called "Storm '93' for studies on extreme precipitation events. Thus, as part of these various initiatives, the Arno River basin has been monitored during some storm events by the Polar $55 \mathrm{C}$, the radar of the Institute of Atmospheric Physics of the National Research Council (CNR) of Italy jointly operated with the University of Florence in Italy.

The Arno River basin is a mountainous region bounded by the Apennines, which form an arc over the river basin with an average altitude of $1000 \mathrm{~m}$ above sea level with a maximum altitude of $2000 \mathrm{~m}$. The 
mountainous terrain of the radar location and the coverage area is prone to severe ground-clutter contamination. The applicability of $Z_{\mathrm{DR}}$-based algorithms for rainfall estimation in an operational setting (where routine scans are made at 10 -min intervals over a $36-\mathrm{h}$ period) over the Arno River basin is studied in this paper. The basin is also instrumented with several rain gauges. This study also provides an opportunity to test the robustness of the algorithms used in the presence of ground-clutter contamination and attenuation problems. Two approaches are used, namely: (a) pointwise comparison, where the rainfall at a gauge location is estimated from the radar as a function of time, and (b) comparison in a statistical framework using the cumulative distribution function (CDF) procedure (Calheiros and Zawadzki 1987) in the context of $Z_{\mathrm{DR}}$-based estimation of rainfall. In addition, the CDF matching technique is utilized to obtain the parameters of $Z_{\mathrm{DR}^{-}}$ based algorithm.

It is well known that radar and rain gauges go through fundamentally different processes to estimate rain, Rain gauges collect the raindrops over a period of time to estimate rainfall, whereas radar obtains instantaneous snapshots of electromagnetic backscatter from a rain volume that is then converted to rainfall via some algorithms. Both instruments have their advantages and disadvantages, such as,

(a) rain gauge measurements are made over a very small area compared to those obtained with radars,

(b) rain gauge measurements have to be integrated over some finite time interval to get a good estimate of rainfall,

(c) radar measurements have to go through an algorithm conversion to estimate rainfall.

Spatial and temporal averaging of radar and rain gauge data has always been used by researchers to reduce the discrepancy between radar and rain gauge estimates of rainfall. Therefore, extensive analysis of space-time averaging of rainfall over the basin is conducted to study the error structure of the comparison between the radar and the gauges.

Our paper is organized as follows. Section 2 briefly presents the principle of rainfall estimation at $\mathrm{C}$ band. In section 3 the radar and rain gauge datasets used in this research work are described. Section 4 presents the pointwise comparison of radar and rain gauges, as well as the probability matching procedure using multiparameter algorithms. In section 5 the analysis of the space-time averaging procedure over the basin is presented. Section 6 summarizes the key results of this paper.

\section{Rainfall estimates at $\mathbf{C}$ band}

The distribution of raindrop size and shape forms the building block for obtaining the properties of the rain medium such as the reflectivity $Z$, rainfall rate $R$, and the differential reflectivity $Z_{\mathrm{DR}}$. The gamma distribution model can adequately describe many of natural variations in the raindrop size distribution (RSD) (U1brich 1983). This model is given by

$$
N(D)=N_{0} D^{\mu} \exp \left[\frac{-(3.67+\mu) D}{D_{0}}\right],
$$

where $N_{0}, D_{0}$, and $\mu$ are the parameters of the RSD. Rainfall rate $R$ and the radar parameters such as $Z_{H}$ and $Z_{\mathrm{DR}}$ can be expressed in terms of the RSD as follows:

$$
R=0.6 \pi \times 10^{-3} \int D^{3} N(D) v(D) d D,
$$

where $v(D)$ is the terminal fallspeed in still air;

$$
\begin{gathered}
Z_{H, V}=\frac{\lambda^{4}}{\pi^{5}|K|^{2}} \int \sigma_{H, V}(D) N(D) d D \\
Z_{\mathrm{DR}}=10 \log \left(\frac{Z_{H}}{Z_{V}}\right)
\end{gathered}
$$

(Seliga and Bringi 1976).

Utilizing the radar observables $Z_{H}$ and $Z_{\mathrm{DR}}$, two estimates of rainfall rate $R$ can be obtained as follows (Gorgucci et al. 1994):

$$
\begin{gathered}
R_{Z H}=C_{Z H} Z_{H}^{\nu} \\
R_{\mathrm{DR}}=C_{\mathrm{DR}} Z_{H}^{\alpha} 10^{\beta Z_{\mathrm{DR}},}
\end{gathered}
$$

where $C_{Z H}, C_{\mathrm{DR}}, \nu, \alpha$, and $\beta$ depend on the operating wavelength. Gorgucci et al. (1994) have derived the dual-polarization rainfall algorithm at $\mathrm{C}$ band based on simulation as

$$
R_{\mathrm{DR}}=7.60 \times 10^{-3} Z_{H}^{0.93} 10^{-0.281 Z_{\mathrm{DR}}} .
$$

However, the same simulation can be used to get a representative $Z-R$ relation, and it is given by

$$
R_{Z H}=2.71 \times 10^{-2} Z_{H}^{0.71} \text {. }
$$

The algorithms given by (8) and (9), as well as the Marshall-Palmer rainfall algorithm,

$$
R_{\mathrm{MP}}=3.65 \times 10^{-2} Z_{H}^{0.625},
$$

are the three algorithms used throughout the various procedures in this paper.

\section{Data description}

The radar Polar 55C is located at Montagnana near Florence to provide good radar coverage over the Amo River basin. Figure 1 shows the regional map with the radar location. The Polar 55C is a dual-polarized pencil beam weather radar with a $0.9^{\circ}$ beamwidth. The radar signals are processed by a SP20 radar signal processor (manufactured by Lassen Research) that is capable of obtaining real-time estimates of reflectivity at horizontal polarization $Z_{H}$ and the differential reflectivity $Z_{\mathrm{DR}}$. More details about the radar can be found in Scarchilli 




FIG. 1. The location of Polar 55C radar.

et al. (1991). The Arno River basin was also instrumented with a network of 41 tipping-bucket rain gauges. The rain gauge network was operated by the "Servizio Idrografico e Mareografico" of Pisa, Italy. The rainfall accumulation in the rain gauges was recorded every $15 \mathrm{~min}$ with a resolution of $0.2 \mathrm{~mm}$. The rain gauges were distributed throughout the basin, with the closest being $12 \mathrm{~km}$ and the farthest being $90 \mathrm{~km}$ from the radar. The experimental region is a mountainous terrain, and therefore the gauges were at various altitudes ranging between sea level and $1400 \mathrm{~m}$. Figure 2 shows the location and the altitude of the rain gauges with respect to the radar.

The data presented in this paper were collected during a meteorological event that occurred on 30 and 31 October 1992 over central Italy. Radar and rain gauge data were collected during this event covering a time period of $36 \mathrm{~h}$. The event was associated with the passage of a frontal perturbation that originated on the southern Mediterranean areas and moved toward north, northeast; that was characterized by the presence of very unstable masses of warm moist air. The storm associated with this event produced intense rainfall over the Amo River basin, creating flood warnings in some of the rivers in the Arno River basin. During this event the Polar 55C was put on an "operational mode" to monitor the basin for hydrological application. This mode consisted of a scan strategy as follows. PPI scans were done over full $360^{\circ}$ in azimuth at a fixed elevation angle at routine time intervals. Because of the excessive ground clutter in the area, the elevation angle was chosen to be $1.8^{\circ}$. The melting layer of the storm was at $3.5 \mathrm{~km}$, and therefore most of the radar measurements were in the rain phase of the storms. The time interval between the PPI scans was set to be 10 min to sample the storm system adequately. Figure 3 shows a typical PPI of the reflectivity data from the storm. The radar measurements were obtained integrating 64 sample pairs of the radar returns with a pulse repetition time (PRT) of $0.85 \mathrm{~ms}$. The archived parameters were the reflectivity at horizontal polarization, the differential reflectivity, the mean Doppler velocity, and spectral width.

Several preprocessing data reduction procedures were applied to the radar data as described below. The radar reflectivity was thresholded at $-10 \mathrm{~dB} Z$ to avoid possible noise contamination. This procedure can potentially remove good data close to the radar, where the -10-dBZ levels could be above noise. However, the regions of the storm with these reflectivity levels do not contribute significantly to rainfall and therefore can be ignored for our application. Second, potential contamination from hail/ice regions were eliminated thresholding on $Z_{\mathrm{DR}}$ values of $0 \mathrm{~dB}$, and enforcing an upper limit of $55 \mathrm{dBZ}$ for the reflectivity factor (Aydin et al. 1986). Again here the loss of good data points near this boundary is outweighed by potentially erroneous data that can bias the rainfall estimates significantly. 


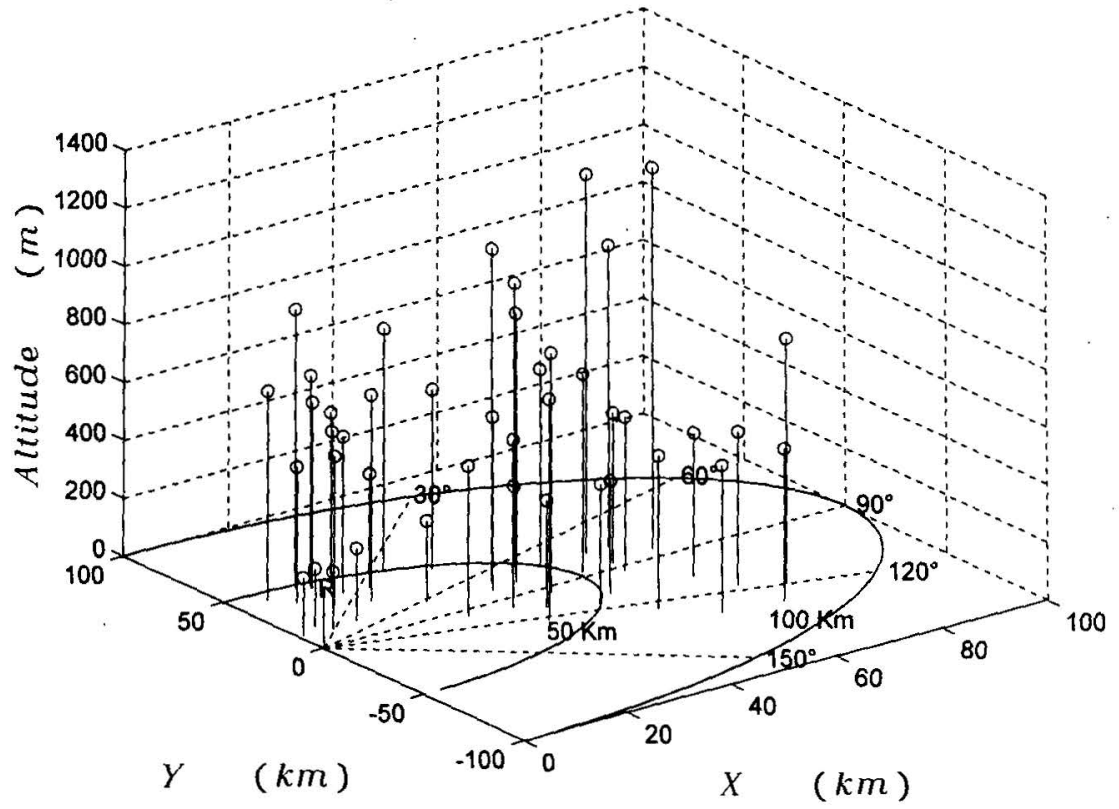

FIG. 2. The location and altitude of the rain gauges with respect to the radar. The radar is in the origin $(0,0)$.

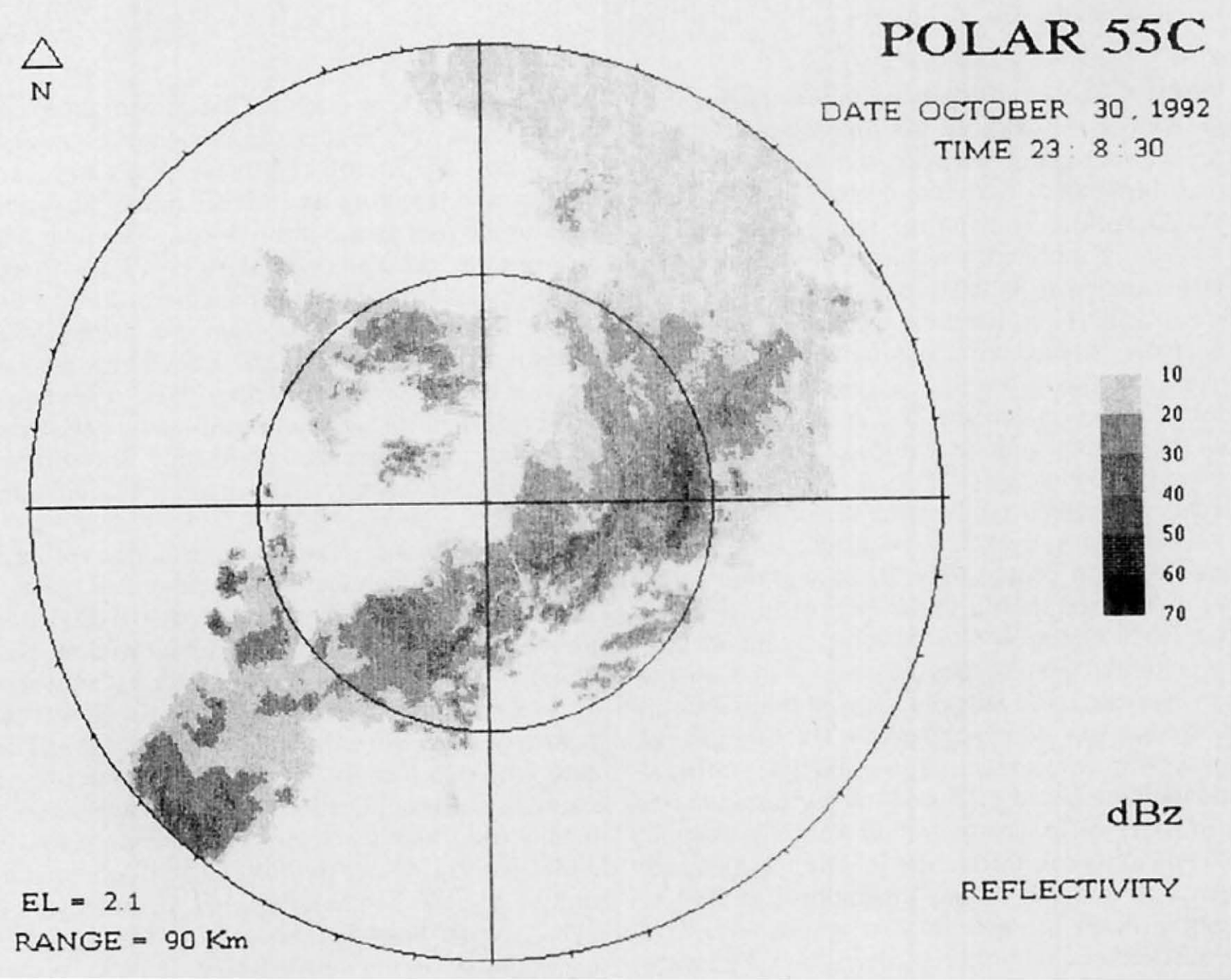

FIG. 3. PPI sample of reflectivity factor. 
Third, potential ground-clutter contamination was removed by eliminating data points with zero velocity and near-zero spectral width, especially at close ranges from the radar. Subsequently, the radar measurements were averaged over nearest neighbors of $1 \mathrm{~km}$ on either side to reduce the measurement error fluctuations.

Radar PPIs are obtained nearly instantaneously, whereas rain gauge data are obtained as accumulation over finite time intervals. With a scan rate of $6^{\circ} \mathrm{s}^{-1}$ it takes 1 min to get a PPI, whereas the rain gauge data in this dataset is integrated over $15 \mathrm{~min}$. Therefore, to enable proper comparison between radar and rain gauge data, the following procedure is adopted: a time series of radar data was constructed at the gauge locations from the instantaneous snapshots of the PPIs, and then this time series was interpolated to provide the time synchronization between the radar and rain gauge data. Figure 4 shows a sample time series of rainfall constructed for a rain gauge and the corresponding radar estimate $R_{Z H}$ at the gauge location.

One additional point of concern with $C$-band radar data in rain is attenuation. It is well established that $C$ band radar signals undergo nonnegligible attenuation and differential attenuation in rainfall. Thus, to be able to use the data successfully, it should be corrected for attenuation. Correction for attenuation can be done either from reflectivity and $Z_{\mathrm{DR}}$ (Aydin et al. 1986) or using differential propagation phase (Bringi et al. 1990; Scarchilli et al. 1993), depending upon the measurement parameters that are available. A cumulative attenuation correction procedure based on $Z_{H}$ and $Z_{\mathrm{DR}}$ is applied here (Gorgucci et al. 1995a). This procedure is a simplified version of the technique suggested by Aydin et al. (1989) and is described in the appendix.

\section{Pointwise radar and rain gauges comparison}

\section{a. Data analysis procedure}

The procedure to compare point rainfall using radar and rain gauges is conceptually straightforward but numerous detail are important. The steps involved in processing radar data for comparison with rain gauge are the following ones.

(a) The location of each rain gauge is mapped onto the radar PPI of reflectivity factor and differential reflectivity. Subsequently, the radar data is converted to rainfall rate using each of the following three algorithms: (i) the Marshall-Palmer relation, (ii) the $Z-R$ relation given by (9), and (iii) the dual-polarization algorithm given by (8).

(b) The radar estimates of rainfall are then averaged over its nearest neighbors of $1 \mathrm{~km}$ either side to obtain average measurements. This is done to smooth the data over measurement errors. The rainfall obtained from the radar over time is then integrated either in time or space, depending on the objectives, and the results are discussed in the following section. An error measure is

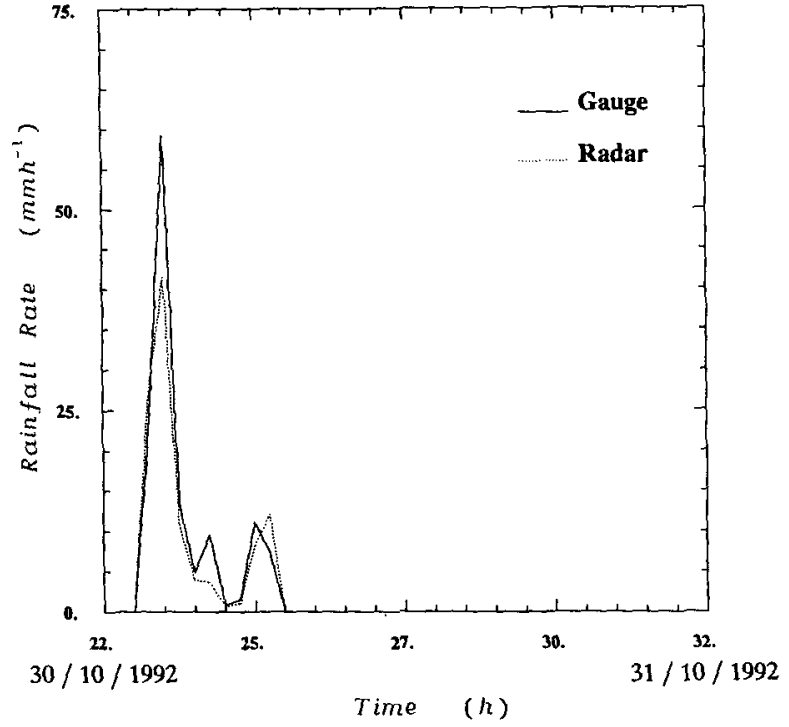

FIG. 4. Time series of rainfall rate constructed from radar and rain gauge measurements. The radar rainfall is obtained using $Z-R$ algorithm.

computed to enable comparison of the three algorithms used in the paper. The estimate of the error measure, namely, the fractional standard error (FSE), can be obtained as

$\mathrm{FSE}=\frac{\left\langle[\text { rainfall }(\text { radar })-\operatorname{rainfall}(\text { gauge })]^{2}\right\rangle^{0.5}}{\langle\text { rainfall }(\text { gauge })\rangle}$,

where the angle brackets indicate the expected value.

\section{b. Experimental results}

Rainfall accumulation at each rain gauge location is estimated from radar measurements for comparison with rain gauge estimates. The FSE of the Marshall-Palmer rainfall estimate was found to be $84 \%$, whereas the standard error in $Z-R$ relation from (9) was found to be $64 \%$. The corresponding standard error in dual-polarization rainfall estimate was $59 \%$. The FSE estimates were also computed for each gauge location, and they were stratified with respect to range from the radar. There was no noticeable trend in the FSE with respect to distance from the radar.

\section{CDF matching analysis}

It can be seen from the pointwise analysis of the previous section that the multiparameter algorithm performs better than the Marshall-Palmer relation as well as the $Z-R$ algorithm given by (9). In this section the $Z-R$ relations and multiparameter algorithms that are obtained by matching the experimental CDF of rainfall 



derived by the radar and rain gauge are analyzed. The CDF of rainfall can be constructed from radar data, and the functional shape of the CDF will depend on the type of algorithm that is used to convert the radar observa-

TABLE 1. The summary of the fractional standard errors FSEs of the pointwise comparison for the various algorithms.

\begin{tabular}{ll}
\hline & FSE \\
\hline$R_{\mathrm{MP}}$ & $84 \%$ \\
$R_{\mathrm{ZH}}$ & $64 \%$ \\
$R_{\mathrm{DR}}(\mathrm{CDF})$ & $59 \%$ \\
$R_{Z H}(\mathrm{CDF})$ & $66 \%$ \\
$R_{\mathrm{DR}}(\mathrm{CDF})$ & $53 \%$ \\
\hline
\end{tabular}



FIG. 5. (a) Experimental cumulative distribution function of rainfall. The solid line shows the CDF observed by the rain gauge, whereas the dotted line shows the CDF obtained from the radar data using Marshall-Palmer relation. (b) Same as (a) except the radar based CDF of rainfall is obtained using $Z-R$ algorithm Eq. (9). (c) Same as (a) except the radar based CDF of rainfall is obtained using the dual-polarization algorithm Eq. (8). tions to rainfall. Figures $5 a-c$ show comparisons of the $C D F$ of rainfall obtained from rain gauge and radar using Marshall-Palmer relation, $Z-R$ relation from (9), and multiparameter algorithm given by (8), respectively. The corresponding error between the two CDFs in self-consistent scale is as follows:

(a) Marshall-Palmer equation, $\mathrm{SSE}=930$,

(b) $Z-R$ equation, $\mathrm{SSE}=317$,

(c) multiparameter rainfall algorithm (8), SSE $=253$,

where SSE is the sum of square errors between the radar-based CDF and rain-gauge-based CDF, obtained as integration of the square deviation between the two $\mathrm{CDFs}$ over the entire range. In practice, the SSE is com- 
puted as the numerical integration of the square of the deviation between the two CDF curves.

The above analysis based on CDF of rainfall rate suggests that without knowledge of the right kind of $Z-R$ relations to use, multiparameter radar rainfall algorithm can match the natural CDF of rainfall better than the $Z-R$ relations considered here.

The optimum $Z-R$ and the multiparameter relations can be obtained matching the CDFs. The rainfall conversion algorithm can be $Z-R$ based or multiparameter based depending on the algorithm used. The $Z-R$ algorithms have parameters of the following form (5):

$$
R\left(Z_{H}\right)=C_{Z H} Z_{H}^{\prime},
$$

where $C_{Z H}$ and $\nu$ are parameters of the algorithm. Similarly, the multiparameter rainfall estimate $R\left(Z_{H}, Z_{\mathrm{DR}}\right)$ has the form (6)

$$
R\left(Z_{H}, Z_{\mathrm{DR}}\right)=C_{\mathrm{DR}} Z_{H}^{\alpha} 10^{-\beta Z_{\mathrm{DR}}},
$$

where $C_{\mathrm{DR}}, \alpha$, and $\beta$ are the parameters of the algorithm. The procedure for estimating the parameters of the radar rainfall algorithm based on CDF matching is as follows:

(i) For a given starting guess of parameters $C_{Z H}, \nu$, $C_{\mathrm{DR}}, \alpha$, and $\beta$ evaluate the radar rainfall estimate at each rain gauge location as described in section 4. Typically, the initial guess is based on established relation such as Marshall-Palmer or multiparameter algorithm given by ( 8 ).

(ii) Construct the CDF based on result of step (i).

(iii) Construct the CDF based on rain gauge observations.

(iv) Construct the SSE.

(v) Iterate the coefficients to minimize the meansquare error.

It should be here noted that the optimization is done using nonlinear algorithms to minimize the SSE. Neither the $R\left(Z_{H}\right)$ relation nor the $R\left(Z_{H}, Z_{\mathrm{DR}}\right)$ is linearized taking logarithms. The linearization procedure taking logarithms disturbs the natural distribution of rainfall in the minimization process. The resulting parameter estimates for $R\left(Z_{H}\right)$ and $R\left(Z_{H}, Z_{\mathrm{DR}}\right)$ based on $\mathrm{CDF}$ matching criteria are as follows:

$$
R_{Z H}(\mathrm{CDF})=4.07 \times 10^{-2} Z_{H}^{0.71},
$$

with the corresponding $\mathrm{SSE}=130$, and

$$
R_{\mathrm{DR}}(\mathrm{CDF})=1.80 \times 10^{-2} Z_{H}^{0.88} 10^{-0.374 Z_{\mathrm{DR}}},
$$

with the corresponding SSE $=108$.

One important observation that can be made from the results depicted in (12) and (13) is that the best multiparameter algorithm has a lower sum square error compared to the best $Z-R$ relation, thereby indicating that even in a statistical approach to rainfall estimation, polarimetric techniques have some improvement to offer. It is to be noted here that a new approach is used to obtain the parameters of dual-polarization radar estimates of rainfall. The algorithms given by (12) and (13) have been obtained in a statistical framework for radar-rain gauge comparison. The rainfall accumulation at the gauge locations based on (12) and (13) is then compared using the procedure similar to the analysis done in section 4. Table 1 shows the summary of pointwise comparison of the various algorithms and the associated FSEs. The total standard error based on $R_{Z H}(\mathrm{CDF})$ is $66 \%$, whereas the corresponding standard error for $R_{\mathrm{DR}}(\mathrm{CDF})$ is $53 \%$. Though the parameterizations (12) and (13), which are obtained through the optimization criteria, are different from the equations (8) and (9), the results are similar, that is, $R_{\mathrm{DR}}(\mathrm{CDF})$ performs slightly better than $R_{Z H}(\mathrm{CDF})$.

\section{Space-time averaging of rainfall estimates from radar and rain gauge}

In this section a comparative study of spatial and temporal averaging of rainfall estimates is presented. To be able to conduct meaningful spatial averages, a subset of the gauges located close to each other are used, so that spatial averaging over the small area will not have large gradients in rainfall rates. Spatial and temporal averaging typically reduces the discrepancy in rainfall comparison due to several reasons. The discrepancy in the rainfall volumes sampled by radar and rain gauges progressively decreases as the samples in space or time are increased. However, to conduct an error analysis of space-time averages is not easy because as soon as any averaging is done, the sample size reduces by the same amount. In this section an error evaluation of the spatial and temporal averaging over a small area is presented. The location of the small basin is shown in Fig. 6.

The FSE evaluation of spatial and temporal averaging is done as follows:

(a) The time series data from the several gauges in the basin, as well as the corresponding radar data, are arranged in a matrix.

(b) The data are then averaged over the nearby gauges or consecutive time intervals to obtain spatially averaged estimates or temporally averaged estimates, where the extent of averaging (or the number of units averaged) is the same.

(c) Subsequently, the averaged estimates are used in the FSE analysis.

Figures 7 and 8 show that the FSE of rainfall for the various algorithms as a function of the number of units of temporal and spatial averaging, respectively. For example, $n$ units of spatial averaging correspond to $n$ rain gauges nearby, whereas the same $n$ units of temporal averaging correspond to consecutive averaging over $n$ rain gauge measurements, where each rain gauge estimate is an accumulation over $15 \mathrm{~min}$. The radar rainfall estimates and rain gauge data were averaged in iden- 


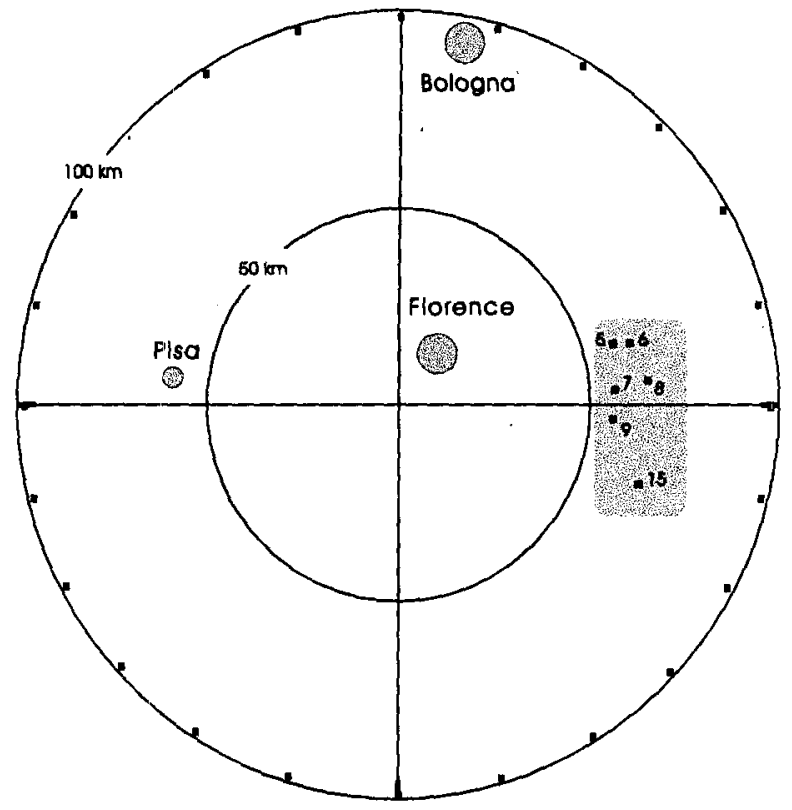

FIG. 6. Location of small basin.

tical ways. Figure 7 shows the FSE results of the five rainfall algorithms as a function of the number of units of temporal averaging. The various algorithms are as follows: (i) $R_{\mathrm{MP}}$ the Marshall and Palmer algorithm (10), (ii) $R_{\mathrm{DR}}$ algorithm from (8), (iii) $R_{Z H}$ algorithm from (9), (iv) $R_{Z H}$ (CDF) algorithm from (12) and, (v) $R_{\mathrm{DR}}(\mathrm{CDF})$ algorithm from (13). It can be seen from the results of Fig. 7 that the algorithm $R_{\mathrm{DR}}$ performs better than $R_{Z H}$ and $R_{\mathrm{MP}}$. In addition the $R_{Z H}(\mathrm{CDF})$ performs as well as $R_{\mathrm{DR}}$. However, $R_{\mathrm{DR}}$ (CDF) performs the best among the five algorithms. It should be noted here that the analysis in this section involves only the data from a smaller basin and, therefore, the analysis does not use the exact dataset that was used to obtain the CDF matching. One observation that can be made from these error quantities is that in a routine operational scenario, $R_{\mathrm{DR}}$ does not perform significantly better than the best $Z-R$ relation. However, without knowledge of the best $Z-R$ relation to use, $R_{\mathrm{DR}}$ still outperforms an arbitrary $Z-R$ relation. Figure 8 shows results similar to Fig. 7 except that the averaging is in spatial domain. Figure 8 shows the FSE of the five algorithms, namely, $R_{\mathrm{MP}}, R_{Z H}, R_{\mathrm{DR}}, R_{Z H}(\mathrm{CDF})$, and $R_{\mathrm{DR}}(\mathrm{CDF})$ with respect to units of spatial averaging. The comparison between the five algorithms is similar to that of Fig. 7. From the above discussion, the comparison of different algorithms with respect to spatial or temporal averaging can be studied; it should be noted here that there is no relation between the units of spatial and temporal averaging. A comparison of the same algorithm between Figs. 7 and 8 shows that the FSE decreases more sharply with temporal averaging in comparison to spatial averaging. This result is due to the

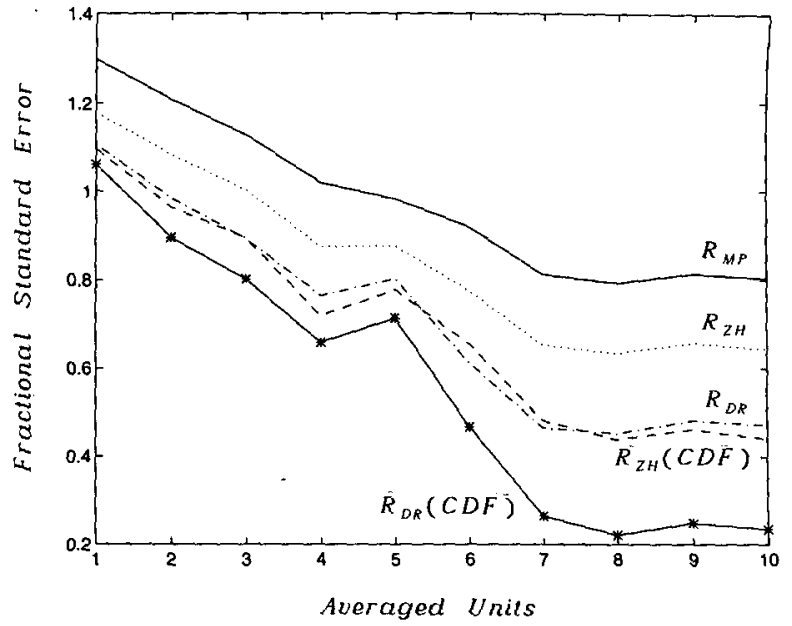

FIG. 7. Fractional standard error of the rainfall for the five algorithms $R_{\mathrm{MP}}, R_{\mathrm{Z} H}, R_{\mathrm{DR}}, R_{Z H}(\mathrm{CDF})$, and $R_{\mathrm{DR}}(\mathrm{CDF})$ shown as a function of the number of units averaged in time. Each unit corresponds to 15 $\min$.

difference between the correlation structure of rainfall in space and in time.

In the following the FSE from the two averaging procedures is stratified with respect to the cumulative rainfall estimates for the five algorithms, thereby providing a common basis for comparison. The FSE of rainfall can be studied as a function of the total rainfall in two ways, namely: (a) via spatial averaging, and (b) via temporal averaging. Figure 9 shows the FSE of the five algorithms as a function of rainfall amounts, for temporal averaging estimates, whereas Fig. 10 shows the same five algorithms as a function of the total rainfall amounts obtained with spatial averaging estimates.



FIG. 8. Fractional standard error of the rainfall for the five algorithms $R_{\mathrm{MP}}, R_{\mathrm{ZH}}, R_{\mathrm{DR}}, R_{\mathrm{Z} H}(\mathrm{CDF})$, and $R_{\mathrm{DR}}(\mathrm{CDF})$ shown as a function of the number of units (gauges) averaged over space. 




FIG. 9. Fractional standard error of the rainfall obtained integrating in time for the five algorithms $R_{\mathrm{MP}}, R_{\mathrm{ZH}}, R_{\mathrm{DR}}, R_{Z H}(\mathrm{CDF})$, and $R_{\mathrm{DR}}(\mathrm{CDF})$ shown as a function of rainfall accumulation.

It should be noted here that Figs. 9 and 10 were also constructed using data from the small basin only. The intercomparison of the algorithms for each case is similar to those of Figs. 7 and 8. With this stratification, it appears that the errors decrease with rainfall amounts, and the error levels are comparable for the same rainfall amount irrespective of the averaging process (spatial or temporal).

\section{Summary and conclusions}

One of the first datasets of its kind, namely, operational monitoring by a dual-polarization radar at $\mathrm{C}$ band over a rain gauge network, is presented here. The applicability of dual-polarization $\left(Z_{\mathrm{DR}}\right)$-based rainfall algorithms in an operational setting is studied. The reflectivity and differential reflectivity measurements from Polar 55C are utilized to obtain estimates of rainfall amounts for an intense precipitation event over the Arno River basin that lasted for a couple of days. The radar was operating in an environment with groundclutter contamination. Since the operating frequency was in $\mathrm{C}$ band, there was also attenuation of the radar signal. The radar data was collected in full $360^{\circ}$ PPIs at routine intervals. Data collected in the above mode was analyzed in two ways, namely:

(a) pointwise comparison of radar and rain gauge estimates,

(b) statistical comparison via probability matching procedure.

Conventionally, multiparameter radar estimates of rainfall have taken the first approach, and the second approach has used only reflectivity. In this paper multiparameter radar data are utilized both ways to study the comparison of radar and rain gauge measurements of rainfall estimates. The fractional standard error defined in the paper was utilized as a quantitative measure of the error in the discrepancy between the radar and rain gauge estimates. Pointwise comparison over the entire event showed that the $R_{\mathrm{MP}}$ had a FSE of $84 \%$ and the $R_{Z H}$ algorithm described in our paper had an FSE of $64 \%$ and the $Z_{\mathrm{DR}}$-based $R_{\mathrm{DR}}$ algorithm had a FSE of $59 \%$.

The experimental CDF obtained from the radar and the rain gauge network were analyzed and compared. This analysis showed that the Marshall-Palmer algorithm $R_{\mathrm{MP}}$ and $R_{Z H}$ have SSE of 930 and 317, respectively, whereas the multiparameter rainfall algorithm using $Z_{\mathrm{DR}}$ has a SSE of 253 . Subsequently parameterizations for the $Z-R$ and $R_{\mathrm{DR}}$ relations were obtained under the criterion that the sum square error between the experimental CDF of rainfall obtained from radar and the rain gauge is minimized. This evaluation showed that $R_{\mathrm{DR}}$ can be parameterized to match the rain gauge $C D F$ better than $Z-R$ relation. This indicates that polarization diversity radar measurements can be used successfully in a statistical framework. The $R_{\mathrm{DR}}(\mathrm{CDF})$ algorithm was also used in pointwise comparison that gives an FSE of $53 \%$.

Averaging in time and/or space is a very commonly used procedure to reduce the discrepancy between radar and rain gauge measurement. The behavior of the fractional standard error as a function of the amount of averaging done in space and time is analyzed, based on pointwise comparison between radar and rain gauges over a smaller area in the basin. For both spatial averages as well as temporal averages the FSE of rainfall decreased steadily as the amount of averaging is increased. Based on the comparison of the algorithms over the two types of averages, we can conclude that (i) $R_{\mathrm{DR}}$ performs better than $R_{\mathrm{MP}}$ and $R_{Z H}$, (ii) the best

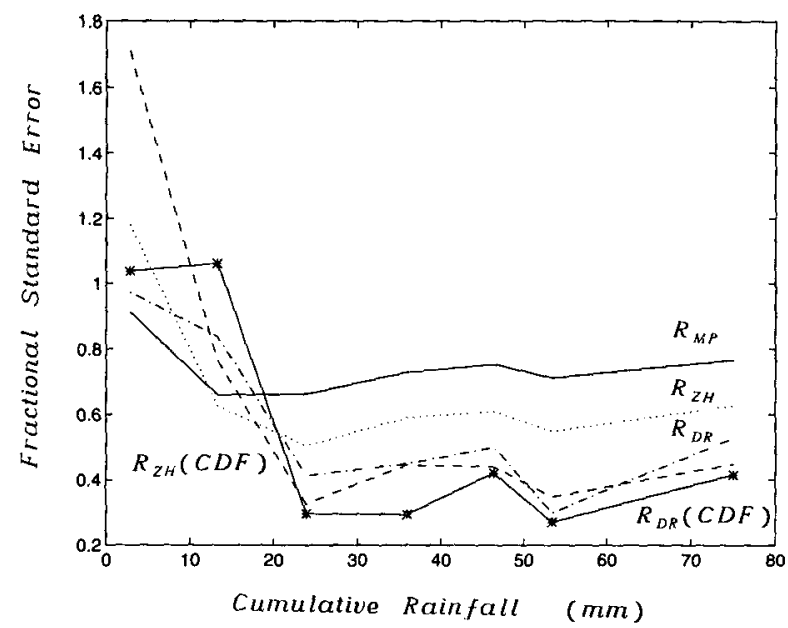

FIG. 10. Fractional standard error of the rainfall obtained integrating over space for the five algorithms $R_{\mathrm{MP}}, R_{Z H}, R_{\mathrm{DR}}, R_{\mathrm{ZH}}(\mathrm{CDF})$, and $R_{\mathrm{DR}}(\mathrm{CDF})$ shown as a function of rainfall accumulation. 
$Z-R$ tuned to the data based on CDF matching algorithm performs as well as the theoretical (ad hoc) $R_{\mathrm{DR}}$ algorithm, and (iii) the CDF-matched $R_{\mathrm{DR}}$ algorithms perform the best among the five algorithms studied. Therefore, on the basis of FSE, SSE, of CDF matching as well as space-time averaging, it appears that in an operational environment with ground-clutter contamination as well as attenuation problems, $R_{\mathrm{DR}}$ cannot perform much better than the best possible $R_{Z H}$. However, without knowledge of the best $Z-R$ relation to use (which may change from storm to storm and region of observation ), $R_{\mathrm{DR}}$ performs better than an arbitrary $Z$ $R$ relation.

Acknowledgments. This research was supported by the EEC program on the Environment "Storm '93", (PL910060: EC-1991-94, IFA-CNR) and by the National Group for Defence from Hydrogeological Hazards (CNR, Italy) and NASA. The authors acknowledge Prof. D. Giuli, Dr. L. Facheris, Dr. L. Baldini, and Dr. E. Palmisano for their collaborative effort in the radar program. The authors would like to offer special thanks to G. Vezzani, A. Volpi, and P. Merendino of SMA, who provided the technical support during the collection of radar data. The authors are grateful to $P$. Iacovelli for assistance rendered during the preparation of the manuscript.

\section{APPENDIX}

\section{Attenuation Correction Procedure}

Reflectivity measurements at $\mathrm{C}$ band are significantly affected by the attenuation of radar signals propagating through the precipitation that exists between the radar and the measurement cell. Differential reflectivity measurements at $\mathrm{C}$ band are similarly affected by the differential attenuation between the horizontal and vertical polarization due to the propagation through the same precipitation path. The absolute specific attenuation $A_{H}$ and the specific differential attenuation $A_{D}$ between the two polarizations are related to RSD as follows (Bringi et al. 1990):

$$
\begin{gathered}
A_{H, V}=4.343 \times 10^{-3} \operatorname{Im} \int_{0}^{\infty} f_{H, V} N(D) d D \\
A_{D}=A_{H}-A_{V},
\end{gathered}
$$

where $f_{H, V}$ are the forward-scattering amplitudes at the two polarization states. Using nonlinear regression analysis, $A_{H}$ and $A_{D}$ can be estimated by means the radar observables $Z_{H}$ and $Z_{\mathrm{DR}}$ as

$$
\begin{aligned}
& \hat{A}_{H}=\alpha_{1} Z_{H}^{\alpha_{2}} 10^{\alpha_{3} Z_{\mathrm{DR}}} \\
& \hat{A}_{D}=\beta_{1} Z_{H}^{\beta_{2}} 10^{\beta_{3} Z_{\mathrm{DR}}} .
\end{aligned}
$$

The coefficients $\alpha_{1}, \alpha_{2}, \alpha_{3}, \beta_{1}, \beta_{2}$, and $\beta_{3}$ can vary with temperature, but not extensively. Based on the en- vironmental conditions, we have used the parameterization at $10^{\circ} \mathrm{C}$. The corresponding coefficients are as follows:

$$
\begin{array}{lll}
\alpha_{1}=6.31 \times 10^{-6}, & \alpha_{2}=0.97, & \alpha_{3}=-0.104 \\
\beta_{1}=5.86 \times 10^{-7}, & \beta_{2}=1.02, & \beta_{3}=-0.030 .
\end{array}
$$

Using a cumulative correction scheme, the corrected value of horizontal reflectivity on decibel scale and differential reflectivity at the $n$th range gate can be estimated as

$$
\begin{gathered}
\left(Z_{H}\right)_{n}=\left(Z_{H}^{\text {meas }}\right)_{n}+\sum_{i=1}^{n-1}\left(\hat{A}_{H}\right)_{i} \Delta r \\
\left(Z_{D R}\right)_{n}=\left(Z_{D R}^{\text {meas }}\right)_{n}+\sum_{i=1}^{n-1}\left(\hat{A}_{D}\right)_{i} \Delta r,
\end{gathered}
$$

where $\Delta r$ is the length $(\mathrm{km})$ of a range gate, $\hat{A}_{H}$ and $\hat{A}_{D}$ are determined from (A3) and (A4), respectively. It should be here noted that some calibration errors in reflectivity factor can drastically deteriorate the estimates corrected for attenuation.

\section{REFERENCES}

Aydin, K., T. A. Seliga, and V. Balaji, 1986: Remote sensing of hail with a dual-linear polarization radar. J. Climate Appl. Meteor., 25, 1475-1484.

- - , Y. Zhao, and T. A. Seliga, 1989: Rain-induced attenuation effects on C-band dual-polarization meteorological radars. IEEE Trans. Geosci. Remote Sens., 27, 57-66.

-_, Y. Lure, and T. A. Seliga, 1990: Polarimetric radar measurements of rainfall compared with ground-based rain gauges during MAYPOLE '84. IEEE Trans. Geosci. Remote Sens., 28, 443-449.

Bringi, V. N., V. Chandrasekar, N. Balakrishnan, and D. S. Zrnić, 1990: An examination of propagation effects in rainfall on radar measurements at microwave frequencies. J. Atmos. Oceanic Technol., 7, 829-840.

Calheiros, R. V., and I. Zawadzki, 1987: Reflectivity-rain rate relationships for radar hydrology in Brazil. J. Climate Appl. Meteor, 26, 118-132.

Gorgucci, E., G. Scarchilli, and V. Chandrasekar, 1994: A robust estimator of rainfall rate using differential reflectivity. J. Atmos. Oceanic Technol., 11, 586-592.

$\ldots$ __ __ and __, 1995a: Radar and rain gauge measurements of rainfall over the Arno basin. Preprints, Conf. on Hydrology, Dallas, TX, Amer. Meteor. Soc., 68-73.

_- , V. Chandrasekar, and G. Scarchilli, 1995b: Radar and surface measurements of rainfall during CaPE. J. Appl. Meteor., 34, $1570-1577$.

Scarchilli, G., E. Gorgucci, D. Giuli, L. Facheris, A. Freni, and G. Vezzani, 1991: Arno project: Radar system and objectives. Preprints, 25th Radar Meteorology Conf., Paris, France, Amer. Meteor. Soc., 805-808.

_ _ _ - V. Chandrasekar, and T. A. Seliga, 1993: Rainfall estimation using polarimetric techniques at C-band frequencies. $J$. Appl. Meteor., 32, 1150-1160.

Seliga, T. A., and V. N. Bringi, 1976: Potential use of the radar reflectivity at orthogonal polarizations for measuring precipitation. J. Appl. Meteor., 15, 69-76.

Ulbrich, C. W., 1983: Natural variations in the analytical form of raindrop size distributions. J. Climate Appl. Meteor., 22, 17641775 . 